\title{
Spin-dependent collision of ultracold metastable atoms
}

\author{
Satoshi Uetake, ${ }^{1,2,{ }^{*}}$ Ryo Murakami, ${ }^{1}$ John M. Doyle, ${ }^{3}$ and Yoshiro Takahashi ${ }^{1,2}$ \\ ${ }^{1}$ Department of Physics, Graduate School of Science, Kyoto University, Kyoto 606-8502, Japan \\ ${ }^{2}$ CREST, Japan Science and Technology Agency, Chiyoda, Tokyo 102-0075, Japan \\ ${ }^{3}$ Department of Physics, Harvard University, Cambridge, Massachusetts 02138, USA
}

(Received 5 June 2012; published 25 September 2012)

\begin{abstract}
Spin-polarized metastable atoms of ultracold ytterbium are trapped at high density and their inelastic collisional properties are measured. We reveal that in collisions of $\mathrm{Yb}\left({ }^{3} P_{2}\right)$ with $\mathrm{Yb}\left({ }^{1} S_{0}\right)$ there is relatively weak inelastic loss, but with a significant spin dependence consistent with Zeeman sublevel changes as being the dominant decay process. This is in strong contrast to our observations of $\mathrm{Yb}\left({ }^{3} P_{2}\right)-\mathrm{Yb}\left({ }^{3} P_{2}\right)$ collisional losses, which are, at low field, much more rapid and have essentially no spin dependence. Our results give a guideline to using the ${ }^{3} P_{2}$ states in many possible applications.
\end{abstract}

DOI: 10.1103/PhysRevA.86.032712

PACS number(s): $34.50 . \mathrm{Cx}, 06.30 . \mathrm{Ft}, 32.70 . \mathrm{Jz}$

\section{INTRODUCTION}

Atoms with alkaline-earth-metal-like electronic structure are under extensive study, partly due to their promise for a number of key applications. For example, the use of long-lived metastable $P$ states (as well as the ground $S$ state) has been explored as a useful quantum computing platform [1-5]. Also, the ultranarrow ${ }^{1} S_{0}-{ }^{3} P_{0}$ atomic resonance in a "magic wavelength" optical lattice may be highly competitive as a new optical frequency standard [6]. In addition, in the area of quantum simulation, there are several theoretical studies of the use of ${ }^{3} P_{J}(J=0,2)$ atoms for studies of Hamiltonians with both spin and orbital degrees of freedom $[7,8]$, implementation of Abelian artificial gauge potentials [9], or simulation of Kondo lattice model [10]. Furthermore, an ytterbium atom, which is a rare-earth-metal atom with an alkaline-earth-metallike electronic structure, has attracted great interest from the viewpoint of fundamental physics since it exhibits a giant parity violation effect [11].

Collisions of metastable $P$-state atoms are interesting not only from a fundamental interactions viewpoint, and as the determining factor in collisional cooling schemes, but also as a crucial mechanism in several key applications. The pioneering work on collisional deexcitation of the metastable $P$ state in atomic $\mathrm{Yb}$ [12] is a good example as it is the key for the successful parity violation experiment [11]. In addition, quantum gate phase imprinting via collisions has been proposed $[2,5,13]$, as well as the exchange interaction in Kondo lattice model simulations [10]. Collisions not only enable new physics but also inhibit desired applications. For example, in optical frequency standards collisional shifts can limit their accuracy, as well as lead to inelastic losses that destroy the atomic sample, possibly on a time scale shorter than the desired interrogation time. Recent studies with metastable alkaline-earth-metal-like structure atoms showed inelastic collision rates as high as $10^{-17}$ to $10^{-16} \mathrm{~m}^{3} / \mathrm{s}$ in ${ }^{3} P_{J}-{ }^{3} P_{J}$ collisions in $\mathrm{Yb}[14]$ and $\mathrm{Sr}[15,16]$ atoms. These

*Present address: Department of Physics, Okayama University, 3-1-1, Tsushima-naka, Kita-ku, Okayama 700-8530, Japan; uetake@scphys.kyoto-u.ac.jp rates, almost as high as the estimated elastic collisional rates, make the use of ${ }^{3} P_{J}$ atoms difficult.

The role of the collisions between the ground $\left({ }^{1} S_{0}\right)$ and excited $\left({ }^{3} P_{J}\right)$ atoms is crucially important in many approaches $[2,5,10]$. In this respect, it is important to investigate the properties of collisions between the metastable triplet states and the ground state $[16,17]$. In addition, the study of anisotropically interacting cold collisions is now a broadening area of study and includes the rare-earth-metal atoms and polar molecules [18-26].

In this article, we report the measurement of the inelastic rate constants for $\mathrm{Yb}$ in both the ${ }^{3} P_{2}-{ }^{3} P_{2}$ and ${ }^{1} S_{0}-{ }^{3} P_{2}$ systems at several magnetic fields below $1 \mathrm{G}$. In particular, we observe strong spin dependence in the inelastic rates for $\mathrm{Yb}\left({ }^{3} P_{2}\right)$ in the ${ }^{1} S_{0}-{ }^{3} P_{2}$ collisional system, with higher energy Zeeman sublevels having higher inelastic rates. This strongly suggests that the inelastic loss is dominated by Zeeman sublevel changing processes ( $m$-changing collisions). These may be induced by the Landau-Zener transition between the entrance $s$-wave channel and the higher partial waves with lower magnetic sublevels [27,28]. In contrast, in the ${ }^{3} P_{2}-{ }^{3} P_{2}$ collisions we observe a much higher inelastic rate that is spin independent. This is consistent with fine structure changing processes $(J$ changing collisions) or principal quantum number changing (PQNC) processes. Our results represent a detailed study of ${ }^{3} P_{2}$ physics; they also provide a road map for the use of the important ${ }^{3} P_{2}$ state and challenge theorists to provide a quantitative explanation of this anisotropic collision physics.

\section{EXPERIMENT}

In a previous experiment [14], we studied ${ }^{3} P_{2}$ collisions via indirect excitation of the ${ }^{3} D_{2}$ state to ${ }^{3} P_{2}$. This led to a spin-unpolarized sample at the relatively high temperature of around $40 \mu \mathrm{K}$. Here we prepare spin-polarized samples at a much lower temperature, below $1 \mu \mathrm{K}$, as described below. The experimental procedure is as follows. $\mathrm{Yb}$ atoms in a thermal beam generated from an oven at $375^{\circ} \mathrm{C}$ are decelerated by a Zeeman slower with a strong ${ }^{1} S_{0}-{ }^{1} P_{1}$ transition at $399 \mathrm{~nm}$, and then are loaded into the intercombination $\left({ }^{1} S_{0}-{ }^{3} P_{1}\right)$ magneto-optical trap (MOT) at $556 \mathrm{~nm}[29,30]$. Figures 1(b)-1(d) show a corresponding energy level diagram of $\mathrm{Yb}$, timing diagram of the experiment, and schematic 
(a)

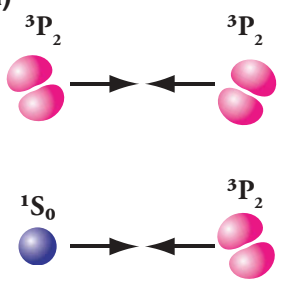

(c)

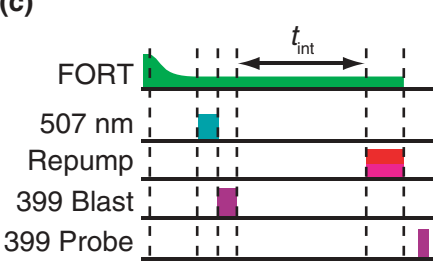

(b)

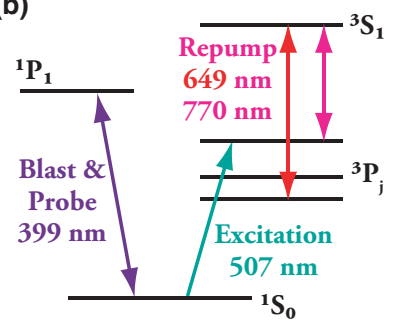

(d)

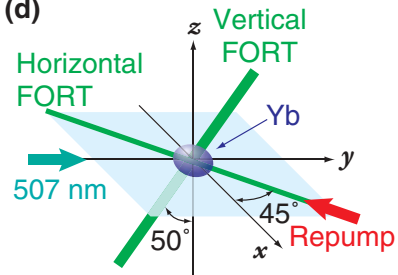

FIG. 1. (Color online) (a) Schematics of the collision measurement. We studied both ${ }^{3} P_{2}-{ }^{3} P_{2}$ and ${ }^{1} S_{0}-{ }^{3} P_{2}$ collisional properties. (b) Energy level diagram of ytterbium. (c) Timing diagram for measurement of collision dynamics of ${ }^{3} P_{2}-{ }^{3} P_{2}$ and ${ }^{1} S_{0}-{ }^{3} P_{2}$ collision system. For measurement of ${ }^{3} P_{2}-{ }^{3} P_{2}$ collision system, unexcited ${ }^{1} S_{0}$ atoms are immediately removed from the trap by applying a strong 399-nm laser. For measurement of ${ }^{1} S_{0}-{ }^{3} P_{2}$ collision, the blast laser is irradiated after interaction time. (d) Schematic diagram of experimental setup. Directions of the 399-nm blast and probe lasers (not shown) are the same as that of 507-nm excitation laser.

diagram of experimental setup, respectively. The atoms are transferred from the MOT to a crossed far-off-resonance trap (FORT) at $532 \mathrm{~nm}$ and evaporatively cooled. The number of atoms is typically $3 \times 10^{5}$. The Bose-Einstein condensation (BEC) transition temperature $T_{c}$ of our trap is $400 \mathrm{nK}$. All the work reported in this paper is done at $480 \mathrm{nK}$, just above $T_{c}$, since we get enough high-atom-number stability for our measurement at this temperature. Our spin-selective excitation employs the very narrow linewidth ${ }^{1} S_{0}-{ }^{3} P_{2}$ transition at $507 \mathrm{~nm}$ $[31,32]$. This transition is a magnetic quadrupole transition and its selection rules are $|\Delta J|=\left|J-J^{\prime}\right|=0,1,2 ; J+J^{\prime} \geqslant 2$, where $J$ and $J^{\prime}$ are the total electronic angular momentum of

the initial and the final states. The excitation efficiency to ${ }^{3} P_{2}$ is about $10 \%$, typically leaving about $2 \times 10^{4}{ }^{3} P_{2}$ atoms in the trap. During the excitation we apply a small bias magnetic field $B_{\text {bias }}$ to spectroscopically split magnetic sublevels $m_{J}$ of ${ }^{3} P_{2}$ state. The applied magnetic field varies from 215 to $848 \mathrm{mG}$, corresponding to the Zeeman splitting of 0.45 to $1.8 \mathrm{MHz}$. The excitation laser linewidth is measured to be less than $1 \mathrm{kHz}$ and Doppler broadening is about $20 \mathrm{kHz}$. This is much less than the Zeeman splitting of $450 \mathrm{kHz}$ at our lowest magnetic field of $215 \mathrm{mG}$, thus ensuring selective creation of spin-polarized samples of atoms in a single Zeeman sublevel.

We study two different collisional systems, ${ }^{3} P_{2}-{ }^{3} P_{2}$ and ${ }^{1} S_{0}-{ }^{3} P_{2}$. For the former, the leftover ${ }^{1} S_{0}$ atoms are immediately removed by strong excitation at $399 \mathrm{~nm}$ (i.e., blast laser) [Fig. 1(c)]. After interaction time $t_{\text {int }}$ (where the ${ }^{3} P_{2}$ atoms collide with other atoms and undergo inelastic loss), ${ }^{3} P_{2}$ atoms are repumped back to the ${ }^{1} S_{0}$ state by 770 - and 649-nm repumping lasers which are resonant to the ${ }^{3} P_{2}-{ }^{3} S_{1}$ and ${ }^{3} P_{0}-{ }^{3} S_{1}$ transitions. A few milliseconds is required for complete repumping. Finally the number of repumped $\left({ }^{1} S_{0}\right)$ atoms is measured by absorption imaging using the ${ }^{1} S_{0}-{ }^{1} P_{1}$ transition. For the latter (i.e., ${ }^{1} S_{0}-{ }^{3} P_{2}$ collision measurement), we simply leave the leftover ${ }^{1} S_{0}$ atoms in the trap. Then the blast laser is irradiated after interaction time: just before the repumping.

\section{RESULTS}

Inelastic atom-atom collisions in various channels are clearly observed for all Zeeman states. A selection of these state-dependent decay curves (those at highest and lowest magnetic fields) are presented in Fig. 2. Figure 2(a) [2(b)] shows the time evolution of the number of ${ }^{3} P_{2}$ atoms without (with) the ${ }^{1} S_{0}$ atoms. Thus the decay of atoms in Fig. 2(a) is due only to ${ }^{3} P_{2}-{ }^{3} P_{2}$ collisions. The measurements reveal a very high inelastic loss rate (approximately the same as the estimated elastic rate) that is essentially independent of both $m_{J}$ and magnetic field strength. This behavior is consistent with the scenario that the dominant decay process is $J$ changing, which (a) ${ }^{3} P_{2}-{ }^{3} P_{2}$ collision system
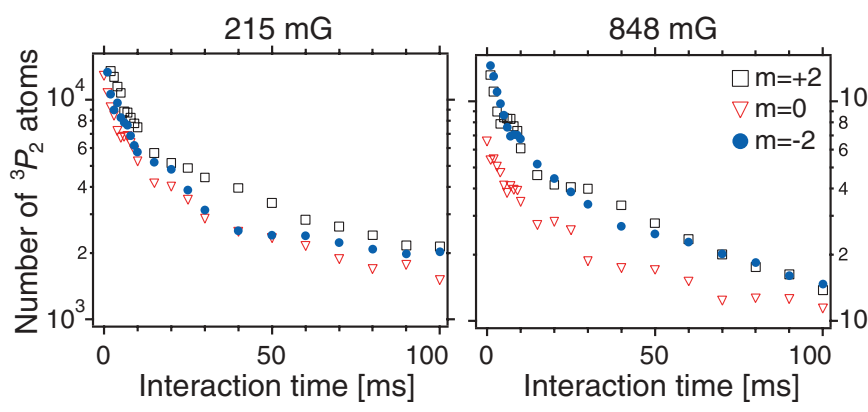

(b) ${ }^{1} S_{0}-{ }^{3} P_{2}$ collision dominant system

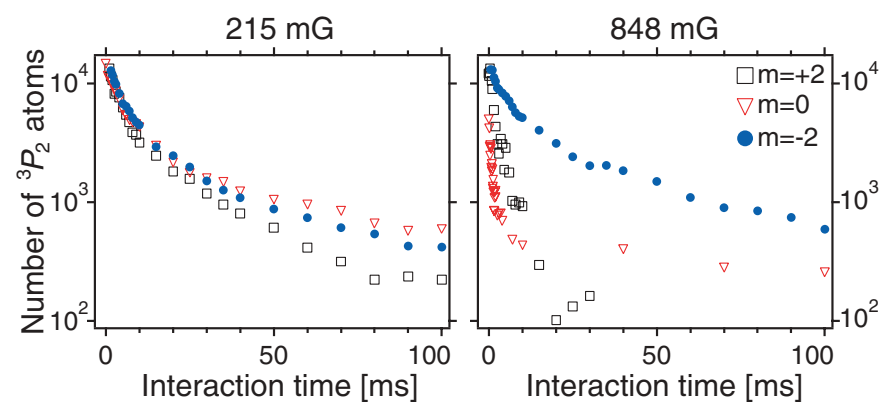

FIG. 2. (Color online) Observed decay of trapped ${ }^{3} P_{2}$ atoms as a function of time. (a) Time evolution of the number of ${ }^{3} P_{2}$ atoms without ${ }^{1} S_{0}$ atoms. Decay rates of different magnetic sublevels $m_{J}$ at different magnetic field strengths are essentially the same. (b) Time evolution of the number of ${ }^{3} P_{2}$ atoms with ${ }^{1} S_{0}$ atoms. In this case the number of ${ }^{1} S_{0}$ atoms is 10 times larger than that of ${ }^{3} P_{2}$ atoms. Thus the ${ }^{3} P_{2}$ atoms dominantly collide with ${ }^{1} S_{0}$ atoms. The behavior is markedly different from that of ${ }^{3} P_{2}-{ }^{3} P_{2}$ collision system. Decay rates are strongly spin dependent. Note that we measured decay for all magnetic components in each bias magnetic field of 215, 307, 407, 596, and 848 mG. Part of the data is shown in these graphs. 


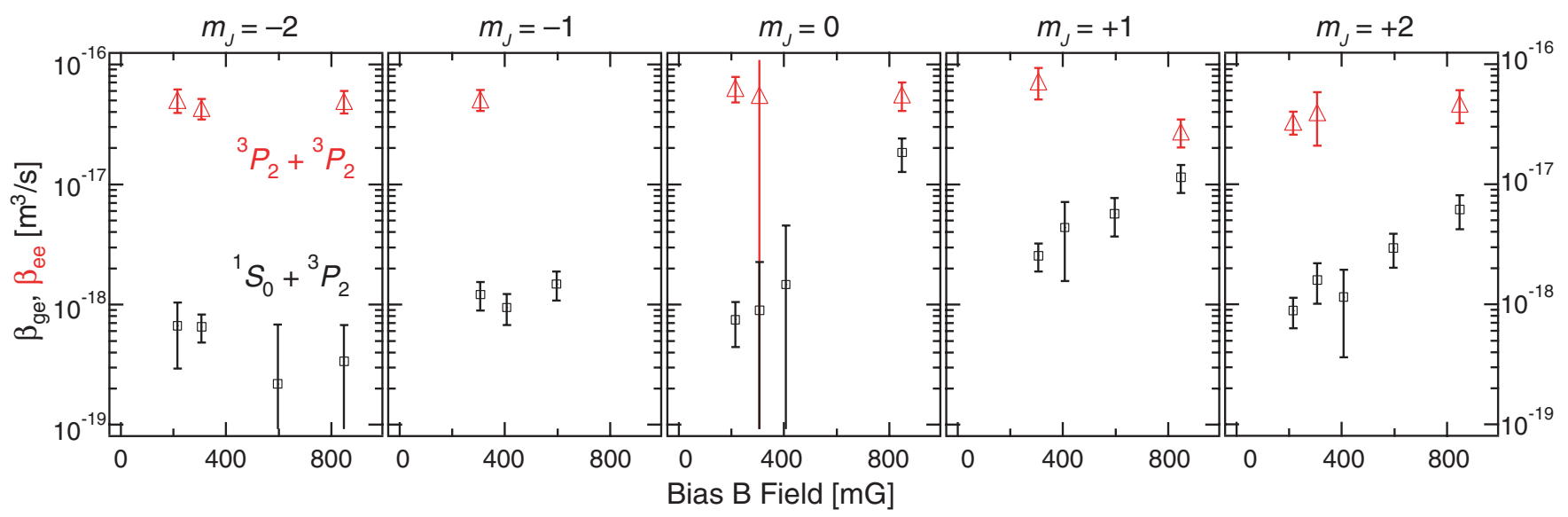

FIG. 3. (Color online) Inelastic decay rates for all $m_{J}$ states in various external magnetic fields. Red triangles show inelastic decay rates $\beta_{e e}^{\text {in }}$ of ${ }^{3} P_{2}-{ }^{3} P_{2}$ collision system. Essentially no spin dependence is observed. Black squares show those of ${ }^{1} S_{0}-{ }^{3} P_{2}$ collision system $\left(\beta_{g e}^{\text {in }}\right)$. Significant spin and magnetic-field dependences are observed.

was inferred by our previous measurement [14]. It may also be due to PQNC collisions, as described in Ref. [15].

The decay of ${ }^{3} P_{2}$ state atoms in collisions dominantly with ${ }^{1} S_{0}$ atoms is shown in Fig. 2(b). It is important to note that in these decay curves the number of ${ }^{1} S_{0}$ atoms is ten times the number of ${ }^{3} P_{2}$ atoms in either Fig. 2(a) or 2(b). Thus, although the decay curves are steeper in these raw data, as will be explained in detail later and shown in Fig. $3,{ }^{1} S_{0}-{ }^{3} P_{2}$ collisions are less inelastic than ${ }^{3} P_{2}-{ }^{3} P_{2}$ collisions. One can easily recognize in Fig. 2(b) that the ${ }^{1} S_{0}-{ }^{3} P_{2}$ collisional decay behavior is markedly different from that of ${ }^{3} P_{2}-{ }^{3} P_{2}$ collisions. The observed decay curves are strongly state dependent. In particular, the atoms in higher Zeeman levels in the ${ }^{3} P_{2}$ state $\left(m_{J} \geqslant 0\right)$ show a stronger field dependence in the decay. This clearly suggests the important role of $m$-changing collisions in the ${ }^{1} S_{0}-{ }^{3} P_{2}$ collision.

We extract two-body inelastic loss rate coefficients from the observed decay rates using the following method. The decay is modeled by coupled differential equations:

$$
\dot{n}_{e}=-\Gamma n_{e}-\beta_{g e} n_{g} n_{e}-\beta_{e e} n_{e}^{2}, \quad \dot{n}_{g}=-\Gamma n_{g}-\beta_{g e} n_{g} n_{e},
$$

where $n$ denotes the local density of atoms, subscript $g(e)$ denotes ground (excited) state, $\beta_{g e}\left(\beta_{e e}\right)$ is the two-body loss coefficient for ground-excited (excited-excited) state collisions. The decay time $1 / \Gamma$ for the ground-state atoms, which is limited by background collisions, is measured to be $30 \mathrm{~s}$. Since this decay time is much longer than the observed decay time, we neglect one-body loss terms $\Gamma n_{g(e)}$ in the following analysis. For the ${ }^{3} P_{2}-{ }^{3} P_{2}$ collision, Eqs. (1) become simple and are solved analytically, since all atoms in the ground state are removed from the trap $\left(n_{g}=0\right)$. By spatially integrating the equation, we obtain the evolution in atom number

$$
N_{e}(t)=\frac{1}{1 / N_{0 e}+G_{e e} t} .
$$

Here $N_{0 e}$ is the initial excited-state atom number and $G_{e e}=\left(V_{2 e} / V_{1 e}^{2}\right) \beta_{e e}$. The effective volume is defined by

$$
V_{q e}=\int d^{3} r\left[n_{e}(\mathbf{r}) / n_{0 \mathrm{e}}\right]^{q},
$$

where $q$ is an integer number, $n_{0 \mathrm{e}}$ is the peak density of excited-state atoms, and $n_{e}(\mathbf{r})$ is the spatial density distribution.

The spatial density distribution depends on the dimensionless parameter $\eta$ : the ratio of the trap depth $\epsilon_{t}$ to the sample temperature $k_{B} T$ in energy units. In our experiment, $\eta$ is 10 for the ground-state atoms. The trap depth for each $m_{J}$ component in the ${ }^{3} P_{2}$ state strongly depends on the direction of the external magnetic field and the polarization of the FORT laser [32,33]. To determine $\eta$ of the ${ }^{3} P_{2}$ state, we measure the Stark shift for all $m_{J}$ states as a function of horizontal FORT laser power in the various bias magnetic fields studied here. The ratio $U_{e} / U_{g}$, where $U_{e(g)}$ is the trap potential of the ${ }^{3} P_{2}\left({ }^{1} S_{0}\right)$ state, varies from 1.02 to 1.31 . From the measurement, $\eta$ for all $m_{J}$ states are calculated to be 7.2 to 11.7 . Since $\eta$ is over 7 for any $m_{J}$ components, a large- $\eta$ approximation can be used to calculate the effective volume. In the large- $\eta$ limit, $n(\mathbf{r})$ is well approximated by the thermal density distribution [34], $n_{e}(\mathbf{r})=$ $n_{0 e} \exp \left[-U(\mathbf{r}) / k_{B} T\right]$, where $U(\mathbf{r})$ is the trap potential. By approximating the FORT potential to a truncated harmonic trap $U(r)=\epsilon_{t}\left(r / R_{0}\right)^{2} \Theta\left(R_{0}-r\right)$, the effective volume can be written as

$$
V_{q}=R_{0}^{3}\left(\frac{\pi}{q \eta}\right)^{3 / 2},
$$

where $\Theta(x)$ is the Heaviside step function, $U\left(R_{0}\right)=\epsilon_{t}$ is the trap depth, and $R_{0}$ is the boundary.

We can extract the $\beta_{g e}$ coefficient by analyzing the decay of ${ }^{3} P_{2}$ atoms in the presence of ${ }^{1} S_{0}$ atoms, shown in Fig. 2(b). The data involve the ${ }^{1} S_{0}-{ }^{3} P_{2}$ collision as well as the ${ }^{3} P_{2}-{ }^{3} P_{2}$. Thus, the analysis is not as simple as Eq. (2), because Eqs. (1) cannot in general be solved analytically. However, if we assume that depletion of the number of ground-state atoms is negligibly small, Eqs. (1) can be solved analytically. In this case, we obtain the atom number evolution in a large- $\eta$ approximation

$$
N_{e}^{\mathrm{A}}(t)=\frac{\exp \left(-G_{g e} N_{0 g} t\right)}{\frac{1}{N_{0 e}}+\frac{1}{N_{0 g}} \frac{G_{e e}}{G_{g e}}\left[1-\exp \left(-G_{g e} N_{0 g} t\right)\right]},
$$

where $G_{g e}=\beta_{g e} /\left(V_{1 e}^{2 / 3}+V_{1 g}^{2 / 3}\right)^{3 / 2}$ and $N_{0 g}$ is the initial ground-state atom number. Note that we can reproduce Eq. (2) from Eq. (5) if we set $N_{0 g}=0$. 
We first fit the ${ }^{3} P_{2}-{ }^{3} P_{2}$ collision data by using Eq. (2) and calculate $\beta_{e e}^{\text {in }}$. Then decay data of ${ }^{1} S_{0}-{ }^{3} P_{2}$ are fitted by using Eq. (5) with fixed $\beta_{e e}^{\text {in }}$. As mentioned in Ref. [35], the observed two-body decay rate $\beta$ includes inelastic collision loss $\beta^{\text {in }}$ and evaporation $\beta^{\text {el }}$ due to elastic collision; i.e., $\beta=\beta^{\text {in }}+f \beta^{\text {el }}$, where $f$ represents the fraction of elastic collisions. The inelastic collision rate can be expressed as $\beta^{\text {in }}=\beta /(f \gamma+1)$, where $\gamma$ is the ratio of elastic to inelastic cross sections, $\gamma \equiv \sigma^{\mathrm{el}} / \sigma^{\text {in }}$ [35]. In a large- $\eta$ approximation, $f$ can be approximated to a simple analytic equation [36]. In the present condition, $\beta^{\text {in }} / \beta$ varies from 0.87 to 0.93 .

The inelastic collision rates for all $m_{J}$ states in various external magnetic fields are plotted in Fig. 3. Essentially no spin dependence in the collision of two metastable ${ }^{3} P_{2}$ states is apparent, which is represented by triangles in Fig. 3. In particular, the spin states of $m_{J}>-2$ show almost the same inelastic collision rates with the lowest energy state of $m_{J}=-2$, which should not suffer from the $m$-changing collision. This behavior is quite different from the significant spin dependence theoretically predicted in the collision of two metastable ${ }^{3} P_{2}$ state at a high magnetic field $[27,28]$. Therefore, our result clearly shows that the $m$-changing collision is not observed for all spin states at this low magnetic field and low temperature. Since the most likely decay process for the $m_{J}=-2$ state is the $J$-changing collision from the ${ }^{3} P_{2}$ state to ${ }^{3} P_{0}$ or ${ }^{3} P_{1}$, this would be dominant. PQNC collision may also contribute to the overall decay. However, it is difficult to distinguish $J$-changing from PQNC collision unless we measure $\beta_{e e}$ for ${ }^{3} P_{0}-{ }^{3} P_{0}$ collision, a task beyond the scope of this paper.

Note that the average of the obtained inelastic collision rate coefficients $\beta_{e e}$ of $4 \times 10^{-17} \mathrm{~m}^{3} / \mathrm{s}$ is higher than the value of $1.0 \times 10^{-17} \mathrm{~m}^{3} / \mathrm{s}$, which was previously obtained at a 100 times higher temperature [14]. Interestingly, an opposite temperature dependence was reported for $\mathrm{Sr}$ atoms $[15,16]$. This interesting temperature dependence is open to further theoretical investigation. In contrast, the significant spin dependence in the collision of the metastable ${ }^{3} P_{2}$ state with the ground state ${ }^{1} S_{0}$ is observed, which is represented by squares in Fig. 3. In particular, significant field dependence is observed for $m_{J}>-1$. Especially at higher fields, the spin states with higher energies $\left(m_{J}>-1\right)$ show higher inelastic collision rates compared with the lowest energy state of $m_{J}=-2$. Since the $m_{J}=-2$ state differs from other spin states only in that the $m_{J}=-2$ state does not suffer from the $m$-changing collision, it is natural to think that the dominant decay process is an $m$-changing collision in this ${ }^{3} P_{2}-{ }^{1} S_{0}$ collision at a low field. The quantitative theoretical explanation of the detailed behaviors is an interesting future work [37].

In addition, we can claim that the $m_{J}=-2$ state is rather stable against the collision with the ${ }^{1} S_{0}$ atom. Since the decay of the ${ }^{3} P_{2}\left(m_{J}=-2\right)$ atoms is dominated by the ${ }^{3} P_{2}-{ }^{3} P_{2}$ collision even in the presence of the ${ }^{1} S_{0}$ atoms, as is shown in Fig. 3, it is quite difficult to accurately extract the $\beta_{g e}$ coefficient for $m_{J}=-2$ state. Although our analysis results in the value of $\beta_{g e}$ on the order of $10^{-19} \mathrm{~m}^{3} / \mathrm{s}$, for the $m_{J}=-2$ state it may be much lower. In fact, our recent measurement on ${ }^{1} S_{0}$ ${ }^{3} P_{2}\left(m_{J}=-2\right)$ atoms in a tightly confined three-dimensional optical lattice indicates that the upper limit of $\beta_{g e}$ is on the order of $10^{-20} \mathrm{~m}^{3} / \mathrm{s}$ [38]. This has promising uses in many applications.

\section{CONCLUSION}

In conclusion, we have experimentally investigated collisional properties of spin-polarized metastable ${ }^{3} P_{2}$ states of $\mathrm{Yb}$ Atoms. We reveal the significant spin dependence in the collision of the metastable ${ }^{3} P_{2}$ state with the ground state ${ }^{1} S_{0}$, which strongly suggests that the dominant decay process is an $m$-changing collision. In contrast, we observe essentially no spin dependence in the collision of two metastable ${ }^{3} P_{2}$ states, which is consistent with the $J$-changing collision as a dominant decay process. Our results will trigger theoretical efforts to clarify these behaviors quantitatively and provide a guideline to using the ${ }^{3} P_{2}$ states in many possible applications.

\section{ACKNOWLEDGMENTS}

The authors acknowledge very helpful experimental assistance of S. Sugawa, S. Kato, and the Yb experiment team in Kyoto university. We also thank T. Tscherbul, P. Zhang, and A. Dalgarno for helpful discussions. This work was supported by the Grant-in-Aid for Scientific Research of JSPS (No. 18204035, No. 21102005C01 (Quantum Cybernetics), and No. 22684022), the GCOE Program "The Next Generation of Physics, Spun from Universality and Emergence" from MEXT of Japan, and FIRST. One of us, J.M.D., thanks the Fulbright program for support.
[1] K. Shibata, S. Kato, A. Yamaguchi, S. Uetake, and Y. Takahashi, Appl. Phys. B 97, 753 (2009).

[2] A. J. Daley, M. M. Boyd, J. Ye, and P. Zoller, Phys. Rev. Lett. 101, 170504 (2008).

[3] R. Stock, N. S. Babcock, M. G. Raizen, and B. C. Sanders, Phys. Rev. A 78, 022301 (2008).

[4] A. Derevianko and C. C. Cannon, Phys. Rev. A 70, 062319 (2004).

[5] A. V. Gorshkov, A. M. Rey, A. J. Daley, M. M. Boyd, J. Ye, P. Zoller, and M. D. Lukin, Phys. Rev. Lett. 102, 110503 (2009).

[6] H. Katori, M. Takamoto, V. G. Pal'chikov, and V. D. Ovsiannikov, Phys. Rev. Lett. 91, 173005 (2003).
[7] A. V. Gorshkov, M. Hermele, V. Gurarie, C. Xu, P. S. Julienne, J. Ye, P. Zoller, E. Demler, M. D. Lukin, and A. M. Rey, Nat. Phys. 6, 289 (2010).

[8] C. Xu, Phys. Rev. B 81, 144431 (2010).

[9] F. Gerbier and J. Dalibard, New J. Phys. 12, 033007 (2010).

[10] M. Foss-Feig, M. Hermele, and A. M. Rey, Phys. Rev. A 81, 051603 (2010).

[11] K. Tsigutkin, D. Dounas-Frazer, A. Family, J. E. Stalnaker, V. V. Yashchuk, and D. Budker, Phys. Rev. Lett. 103, 071601 (2009).

[12] D. F. Kimball, D. Clyde, D. Budker, D. DeMille, S. J. Freedman, S. Rochester, J. E. Stalnaker, and M. Zolotorev, Phys. Rev. A 60, 1103 (1999). 
[13] D. Jaksch, H.-J. Briegel, J. I. Cirac, C. W. Gardiner, and P. Zoller, Phys. Rev. Lett. 82, 1975 (1999).

[14] A. Yamaguchi, S. Uetake, D. Hashimoto, J. M. Doyle, and Y. Takahashi, Phys. Rev. Lett. 101, 233002 (2008).

[15] A. Traverso, R. Chakraborty, Y. N. Martinez de Escobar, P. G. Mickelson, S. B. Nagel, M. Yan, and T. C. Killian, Phys. Rev. A 79, 060702 (2009).

[16] C. Lisdat, J. S. R. Vellore Winfred, T. Middelmann, F. Riehle, and U. Sterr, Phys. Rev. Lett. 103, 090801 (2009).

[17] N. D. Lemke, J. von Stecher, J. A. Sherman, A. M. Rey, C. W. Oates, and A. D. Ludlow, Phys. Rev. Lett. 107, 103902 (2011).

[18] C. I. Hancox, S. C. Doret, M. T. Hummon, R. V. Krems, and J. M. Doyle, Phys. Rev. Lett. 94, 013201 (2005).

[19] R. V. Krems, J. Kłos, M. F. Rode, M. M. Szczęśniak, G. Chałasiński, and A. Dalgarno, Phys. Rev. Lett. 94, 013202 (2005).

[20] M.-J. Lu, V. Singh, and J. D. Weinstein, Phys. Rev. A 79, 050702 (2009).

[21] M. Lu, N. Q. Burdick, S. H. Youn, and B. L. Lev, Phys. Rev. Lett. 107, 190401 (2011).

[22] M. Lu, N. Q. Burdick, and B. L. Lev, Phys. Rev. Lett. 108, 215301 (2012).

[23] S. Kotochigova and A. Petrov, Phys. Chem. Chem. Phys. 13, 19165 (2011).
[24] A. Petrov, E. Tiesinga, and S. Kotochigova, arXiv:1203.4172.

[25] K. Aikawa, A. Frisch, M. Mark, S. Baier, A. Rietzler, R. Grimm, and F. Ferlaino, Phys. Rev. Lett. 108, 210401 (2012).

[26] L. P. Parazzoli, N. J. Fitch, P. S. Żuchowski, J. M. Hutson, and H. J. Lewandowski, Phys. Rev. Lett. 106, 193201 (2011).

[27] V. Kokoouline, R. Santra, and C. H. Greene, Phys. Rev. Lett. 90, 253201 (2003).

[28] R. Santra and C. H. Greene, Phys. Rev. A 67, 062713 (2003).

[29] T. Kuwamoto, K. Honda, Y. Takahashi, and T. Yabuzaki, Phys. Rev. A 60, R745 (1999).

[30] S. Uetake, A. Yamaguchi, S. Kato, and Y. Takahashi, Appl. Phys. B 92, 33 (2008).

[31] A. Yamaguchi, S. Uetake, and Y. Takahashi, Appl. Phys. B 91, 57 (2008).

[32] A. Yamaguchi, S. Uetake, S. Kato, H. Ito, and Y. Takahashi, New J. Phys. 12, 103001 (2010).

[33] T. Ido and H. Katori, Phys. Rev. Lett. 91, 053001 (2003).

[34] O. J. Luiten, M. W. Reynolds, and J. T. M. Walraven, Phys. Rev. A 53, 381 (1996).

[35] R. deCarvalho and J. Doyle, Phys. Rev. A 70, 053409 (2004).

[36] M. Yan, R. Chakraborty, P. G. Mickelson, Y. N. M. de Escobar, and T. C. Killian, Phys. Rev. A 83, 032705 (2011).

[37] T. V. Tscherbul (private communication).

[38] S. Sugawa, S. Kato, S. Uetake, and Y. Takahashi (unpublished). 